\title{
Quantum Fractal Eigenstates
}

\author{
Giulio Casati $^{(a, b, c)}$, Giulio Maspero ${ }^{(a, b, c)}$ and Dima L. Shepelyansky ${ }^{(d,+, *)}$ \\ (a) Università di Milano, sede di Como, Via Lucini 3, 22100 Como, Italy \\ (b) Istituto Nazionale di Fisica della Materia, Unità di Milano, Via Celoria 16, 20133 Milano, Italy \\ ${ }^{(c)}$ Istituto Nazionale di Fisica Nucleare, Sezione di Milano, Via Celoria 16, 20133 Milano, Italy \\ (d) Isaac Newton Institute, 20 Clarkson Rd, Cambridge, CB3 OEH, U.K.
}

(September 25, 2018)

\begin{abstract}
We study quantum chaos in open dynamical systems and show that it is characterized by quantum fractal eigenstates located on the underlying classical strange repeller. The states with longest life times typically reveal a scars structure on the classical fractal set.
\end{abstract}

PACS numbers: 05.45.+b, 03.65.Sq

At present, the structure of phase space for generic classical Hamiltonian systems is well understood both on qualitative and quantitative levels. With the increase of perturbation's parameter the invariant KAM curves are destroyed by the perturbation and are replaced by chaotic regions. These regions grow and become interconnected over the whole phase space. In this situation the phase space exhibits a hierarchical structure of mixed, chaotic and integrable components which continues on smaller and smaller scales of the phase space. At larger perturbations the measure of stability islands decreases until they become negligible for sufficiently strong perturbations. This is a general scenario for the emergence of chaos in classical Hamiltonian systems [1]. The well known model in which such scenario has been studied in detail is the Chirikov standard map [2].

The investigations of the corresponding quantum systems show that the structure of eigenstates is closely related to the properties of classical phase space. In the integrable regime, eigenstates are located on the invariant KAM curves while in the chaotic regime they spread over the whole chaotic component in agreement with the Shnirelman theorem [3]. The structure of eigenstates can be seen in a pictorial way with the help of Wigner function and Husimi distribution [1. This representation allows to see graphically the qualitative change of eigenstates during the transition from integrability to chaos. In the chaotic regime they also allow to see scarred eigenfunctions in which the probability is concentrated near short unstable periodic orbits [5,6]. However in the chaotic regime, the majority of eigenfunctions are ergodic on the energy surface and the energy level statistics is well described by the Random Matrix Theory [7]. Here we assume that the value of the Planck constant is sufficiently small and the effect of dynamical localization does not alter the properties of eigenstates [8].

As a result, in absence of localization, the structure of quantum eigenstates in the regime of Hamiltonian chaos is now well understood and many concrete examples have been studied numerically [5 8] and experimentally 99. Conversely, the chaotic objects with fractal structure, which appear in dissipative classical dynamics such as strange attractors and repellers, were not studied in quantum mechanics. The main problem is that in the quantum case the dissipation is always accompanied by noise and generally one should study the density matrix 10,11. In this way the problem becomes much more complicated than the Hamiltonian case and the fractal structure of strange attractors/repellers had never been seen in quantum eigenstates.

In this paper we study an open chaotic system in which absorption leads to the appearance of a fractal set in the classical phase space (strange repeller) . The quantum dynamics of the model is governed by a nonunitary evolution operator. Such unitary breaking, due to absorption, was widely used in nuclear and mesoscopic physics to describe coupling with continuum and massive leads via open channels [12].

The underlying classical fractal set should affect the quantum dynamics and find its manifestations in the structure of eigenstates. Indeed it is natural to expect that long living eigenstates will be associated with the above strange set on which classical orbits live forever. Therefore the eigenstates associated with this set should strongly influence the scattering process, relaxation and ionization into continuum.

To investigate the quantum fractal eigenstates we choose the kicked rotator model with absorbing boundary conditions which was introduced in [13]. The quantum dynamics is described by the evolution matrix:

$$
\bar{\psi}=\hat{U} \psi=\hat{P} e^{-i T \hat{n}^{2} / 4} e^{-i k \cos \hat{\theta}} e^{-i T \hat{n}^{2} / 4} \psi,
$$

where $\hat{n}=-i \partial / \partial \theta, \hbar=1$ and the operator $\hat{P}$ projects the wave function to the states in the interval $[-N / 2, N / 2]$. The quasiclassical limit corresponds to $k \rightarrow \infty, T \rightarrow 0$ with the chaos parameter $K=k T=$ const.

The classical dynamics is described by the Chirikov standard map [2]:

$$
\bar{n}=n+k \sin \left[\theta+\frac{T n}{2}\right], \bar{\theta}=\theta+\frac{T}{2}(n+\bar{n}) .
$$


In this model all trajectories (and quantum probabilities) leaving the interval $[-N / 2, N / 2]$ are absorbed and never return back. For the classical map the ionization time required for a trajectory to reach diffusively the absorbing boundary is $t_{c} \sim t_{D}=N^{2} / D$ where $D \approx k^{2} / 2$ is the diffusion rate for $K \gg 1$. The independence of $t_{c}$ on $N$ requires $N / k=$ const.

In our study we fixed $N / k=4$ and $K=7$ so that the phase space is completely chaotic with no visible islands. As a result the classical probability $P(t)$ to stay inside the sample $[-N / 2, N / 2]$ decays exponentially for $t>t_{D}: P(t) \sim \exp \left(-\gamma_{c} t\right)$ with $\gamma_{c}=1 / t_{c}=0.10188$. The quantum probability follows closely the classical one up to the quantum relaxation time scale $t_{q} \sim \sqrt{t_{c} / \Delta} 14$ where $\Delta=1 / N$ is the levels spacing.

In the classical case, the orbits which are never ionized and which stay forever inside the sample form a fractal set (strange repeller). To obtain this set we study the evolution of $M=2.2 \cdot 10^{9}$ classical orbits up to $t=100$ map iterations. Initially the orbits are homogeneously distributed in the phase plane. An example of a set of points which are never ionized and form a fractal is shown in Fig.1. The fractal nature is demonstrated by magnification of a small part of the phase space which clearly shows a structure typical of strange attractors/repellers [1, 15]. Similarly to the problem of diffusion in the Lorentz gas [16], the information dimension $d_{1}$ of this set can be expressed via the Lyapunov exponent $\Lambda \approx \ln K / 2$ and the probability decay rate $\gamma_{c} \sim D / N^{2}: d_{1}=2-\gamma_{c} / \Lambda$. In our case with $\gamma_{c} \approx 0.1, \Lambda \approx 1.25$ this gives $d_{1} \approx 1.92$.

In the quantum system, due to absorption at the boundary, all eigenvalues of $\hat{U}$ move inside the unitary circle and can be written as $\lambda=\exp (-i \epsilon)=$ $\exp (-i E-\Gamma / 2)$. The imaginary part of $\epsilon$ determines the decay rate $\Gamma$ of an eigenstate. Due to the symmetry of the $U$-matrix the eigenstates are symmetric or antisymmetric in $n$ and in order to study the statistics of $\Gamma$ 's we restricted ourselves to investigation of symmetric states. The general structure of the distribution of $\Gamma$ 's had been found in [13]. This distribution $d W / d \Gamma$ has a gap for small values of $\Gamma\left(\Gamma<\gamma_{c}\right)$ while for $\Gamma>\gamma_{c}$ it drops according to a power law, $d W / d \Gamma \sim \Gamma^{-\frac{3}{2}}$ for $t_{c}>>1$ [13]. A typical example for $N=12001$ is shown in Fig.2 and confirms the above global structure of the distribution. With the increase of the matrix size $N$, the minimal value of $\Gamma=\Gamma_{\min }$ converges to the classical value $\gamma_{c}$ as it is shown in the inset of Fig.2. The fit of numerical data gives $\Delta \Gamma=\gamma_{c}-\Gamma_{\text {min }} \approx 6.2 \gamma_{c} g^{-\alpha}$ with $\alpha=0.507 \pm 0.037$. Theoretically we expect that, due to fluctuations, $\Delta \Gamma$ should be of the order of the distance $\delta$ between the eigenvalues of $\hat{U}$ in the complex plane. Since most of the $N$ eigenvalues $\epsilon$ are distributed inside the ring of width $\gamma_{c}$, then $\delta \sim\left(\gamma_{c} / N\right)^{1 / 2} \sim 1 / t_{q}$. This gives $\Delta \Gamma \sim \delta \sim 1 / t_{q}$. Therefore, after the fitting of the data in Fig.2, we have

$$
\Delta \Gamma=\gamma_{c}-\Gamma_{\min } \approx 6.2 \gamma_{c} / \sqrt{g}
$$

where $g=\gamma_{c} / \Delta$ is the effective conductance of the system.

To analyze the structure of eigenfunctions in the phase space we used the Husimi function obtained from the Wigner function smoothed in the intervals $\Delta n$ and $\Delta \theta$ $(\Delta n \Delta \theta=1 / 2)$ (see [4]). The ratio $s=\Delta \theta / \Delta n$ was fixed in a way to have optimal resolution. In Fig.1 it was equal to $s=0.0015(a) ; 6.25 \times 10^{-4}(c) ; 2.25 \times 10^{-4}(e)$ and in Fig. 3 it was $s=0.0015(a) ; 0.16(b, c)$. The Husimi functions were constructed from antisymmetric eigenstates since they had the minimal values of $\Gamma$. The comparison between the density distributions in the phase space for the classical and the quantum case can be seen in Fig.1. The quantum Husimi function for the eigenstates with $\Gamma \approx \gamma_{c}$ reproduces very well the fractal structure of the classical strange repeller on very small scales. It shows close agreement between the classical and quantum data for large $N$ corresponding to a small effective Planck constant $\hbar_{\text {eff }}$. Of course, on very small scales comparable with the minimal quantum cell, the quantum density becomes smooth. However, on scales larger than this cell, the fractal structure is obvious: we call such states quantum fractal eigenstates.

It is natural to expect some analogous of Shnirelman theorem [3] for these quantum fractal eigenstates, so that in the quasiclassical limit they will be distributed over the classical fractal set according to the classical measure. The case of Fig. 1 for the states with $\Gamma \approx \gamma_{c}$ confirm such expectations. However, the situation for the states with $\Gamma=\Gamma_{\min }$ is rather different (Fig.3). Indeed, generally, we observe there the appearance of scars on the underlying classical strange repeller, the silhouette of which, in spite of scars, is still clearly seen. The strength of scars grows with decreasing $N$ (increasing $\hbar_{\text {eff }}$ ). However, even for the largest $N=59049$, the probability distributions $f(n)$ projected on the $n$-axis demonstrate an evident difference between the classical and quantum cases (Fig.4). We qualitatively understand this phenomenon in the following way: the quantum state with the minimal value of $\Gamma$ should stay away as far as possible from the absorbing boundaries so that quantum interference should redistribute probability on the classical set and will lead to some scarring around classical trajectories which correspond to short unstable periodic orbits located near the center. Indeed Fig.4 shows a pronounced peak near period two orbits. There is also some correlation between the density of long periodic orbits (Fig.3d) and quantum distributions with not very large $N$ (Fig.3b,c). However, at present, we cannot propose any quantitative explanations of the scarred eigenstate structure.

The complex eigenvalues of the evolution operator $U$ can be considered as some poles of the scattering matrix. Therefore, we can expect that similar quantum fractal eigenstates will appear in the problems of chaotic scat- 
tering in the quasiclassical regime. One of the possible models to study such effects is the three discs problem where the gap in the $\Gamma$-rates has been discussed in [17]. Such quantum fractal eigenstates can be also studied in experiments with chaotic light in micrometer-size droplets [18] where the classical dynamics is governed by a map analogous to the map (2). In conclusion, we have demonstrated that the quantum eigenstates can form a quantum strange repeller. We conjecture that quantum strange attractors, once identified, should have a similar structure.

We thank B.Georgeot for the discussions about the Shnirelman theorem for nonunitary operators and R.Ketzmerick for useful advises in Lanczos approach to the kicked rotator.

+ On leave from Laboratoire de Physique Quantique, UMR C5626 du CNRS, Université Paul Sabatier, F-31062 Toulouse, France

* Also Budker Institute of Nuclear Physics, 630090 Novosibirsk, Russia

[1] A.J.Lichtenberg and M.A.Lieberman, Regular and Chaotic Dynamics, Springer-Verlag, New York (1992).

[2] B. V. Chirikov, Phys. Rep. 52, 263 (1979).

[3] A. I. Shnirelman, Usp. Mat. Nauk. 29, N6, 181 (1974).

[4] S.J. Chang and K.J. Shi, Phys. Rev. A 34, 7 (1986).

[5] E.J. Heller, Phys. Rev. Lett. 53, 1515 (1984).

[6] O.Agam and S.Fishman, Phys. Rev. Lett. 73, 806 (1994).

[7] O. Bohigas, Les Houches Lecture Series 52, Eds. M.-J. Giannoni, A.Voros, and J. Zinn-Justin (North-Holland, Amsterdam, 1991).

[8] G.Casati and B.V. Chirikov, Quantum Chaos, Cambridge University Press, Cambridge (1995).

[9] J. Stein, H.-J. Stöckmann, and U. Stoffregen, Phys. Rev. Lett. 75, 53 (1995).

[10] F.Haake Quantum Signatures of Chaos., (Springer, Berlin 1992).

[11] R.Graham, in Quantum Chaos, Eds. G.Casati, I.Guarneri and U.Smilansky, North-Holland, Amsterdam, 241 (1993).

[12] C. H.Lewenkopf, and H. A. Weidenmüller, Ann. Phys. 212, 53 (1991).
[13] F.Borgonovi, I.Guarneri and D.L.Shepelyansky, Phys. Rev. A, 43, 4517 (1991).

[14] G.Casati, G.Maspero and D.L.Shepelyansky, condmat/9706103

[15] E.Ott , Chaos in Dynamical Systems, Cambridge Univ. Press, N.Y. (1993).

[16] P.Gaspard, G.Nicolis, Phys. Rev. Lett., 65, 1693 (1990).

[17] P.Gaspard, in Quantum Chaos, Eds. G.Casati, I.Guarneri and U.Smilansky, North-Holland, Amsterdam, 307 (1993).

[18] J. U. Nöckel and A. D. Stone, Nature 385, 45 (1997).

FIG. 1. Left column: Husimi function of the quantum fractal eigenstate for $N=59049$ and $\Gamma=0.1080 \approx \gamma_{c}$. Right column: classical strange repeller obtained, from initially homogeneously distributed orbits, after 100 iterations of map (2). The color varies proportionally to the density: from blue for zero density to bright red for maximal density (the color scale is the same for classical and quantum cases on each magnification level). The size of the phase region $(\theta, n)$ is: $0 \leq \theta \leq 2 \pi,-N / 2 \leq n \leq N / 2$ for (a) and (b); $0 \leq \theta \leq 2 \pi / 3,-N / 6 \leq n \leq N / 6$ for (c) and (d); $0 \leq \theta \leq 2 \pi / 9,-N / 18 \leq n \leq N / 18$ for (e) and (f).

FIG. 2. Distribution $d W / d \Gamma$ as a function of $\Gamma$ for $N=12001$. The arrow marks the value of $\gamma_{c}$. The inset shows $\Delta \Gamma=\gamma_{c}-\Gamma_{\min }$ vs. $N$ in log-log scale. The straight line shows the dependence given by (3). To guide the eye, the numerical data (points) are connected by lines.

FIG. 3. Husimi function for quantum eigenstates with minimal $\Gamma=\Gamma_{\min }$ for: (a) $N=59049$; (b) $N=729$ and (c) $N=243$. The case (d) shows the classical initial density of orbits which survive after 60 iterations forward and back in time. The maximum of density (bright red) from (a) to (d) is fixed by case (a).

FIG. 4. Probability distribution $f(n)$ over the unperturbed basis $n$ for quantum eigenstates of Fig.1a (dotted curve), Fig.3a (dashed curve). The full curve is the classical distribution of Fig.1b. Crosses mark periodic orbits with period one at $(0,0)$ and period two at $(n= \pm \pi / T$ for $\theta=\pi / 2,3 \pi / 2)$. The asymmetry of $f(n)$ in $n$ for the classical case is due to round off computer errors. 
This figure "figilab.gif" is available in "gif" format from: http://arxiv.org/ps/cond-mat/9710118v1 
This figure "figilcd.gif" is available in "gif" format from: http://arxiv.org/ps/cond-mat/9710118v1 
This figure "figvlef.gif" is available in "gif" format from: http://arxiv.org/ps/cond-mat/9710118v1 


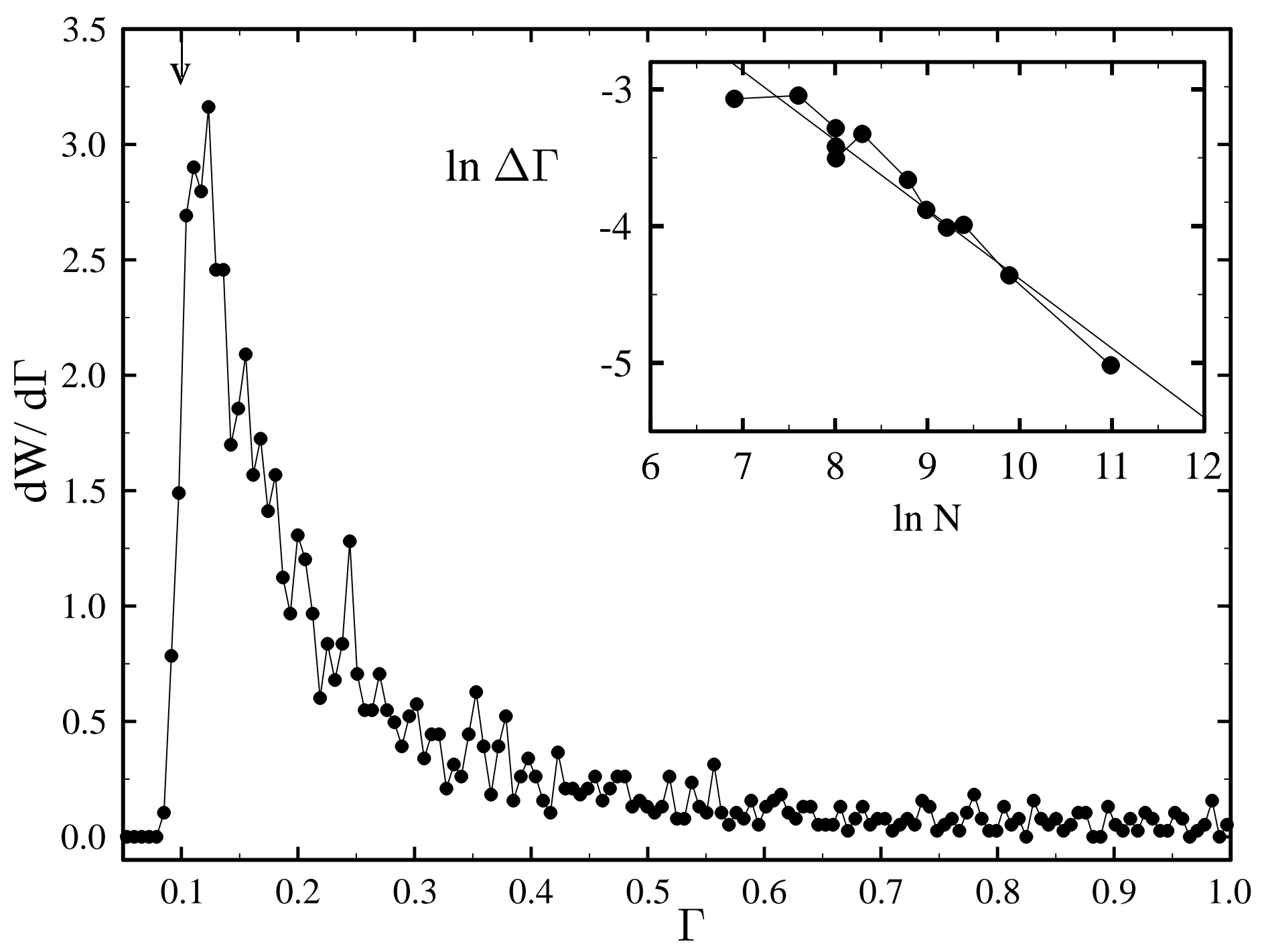


This figure "fig3ab.gif" is available in "gif" format from: http://arxiv.org/ps/cond-mat/9710118v1 
This figure "fig3cd.gif" is available in "gif" format from: http://arxiv.org/ps/cond-mat/9710118v1 


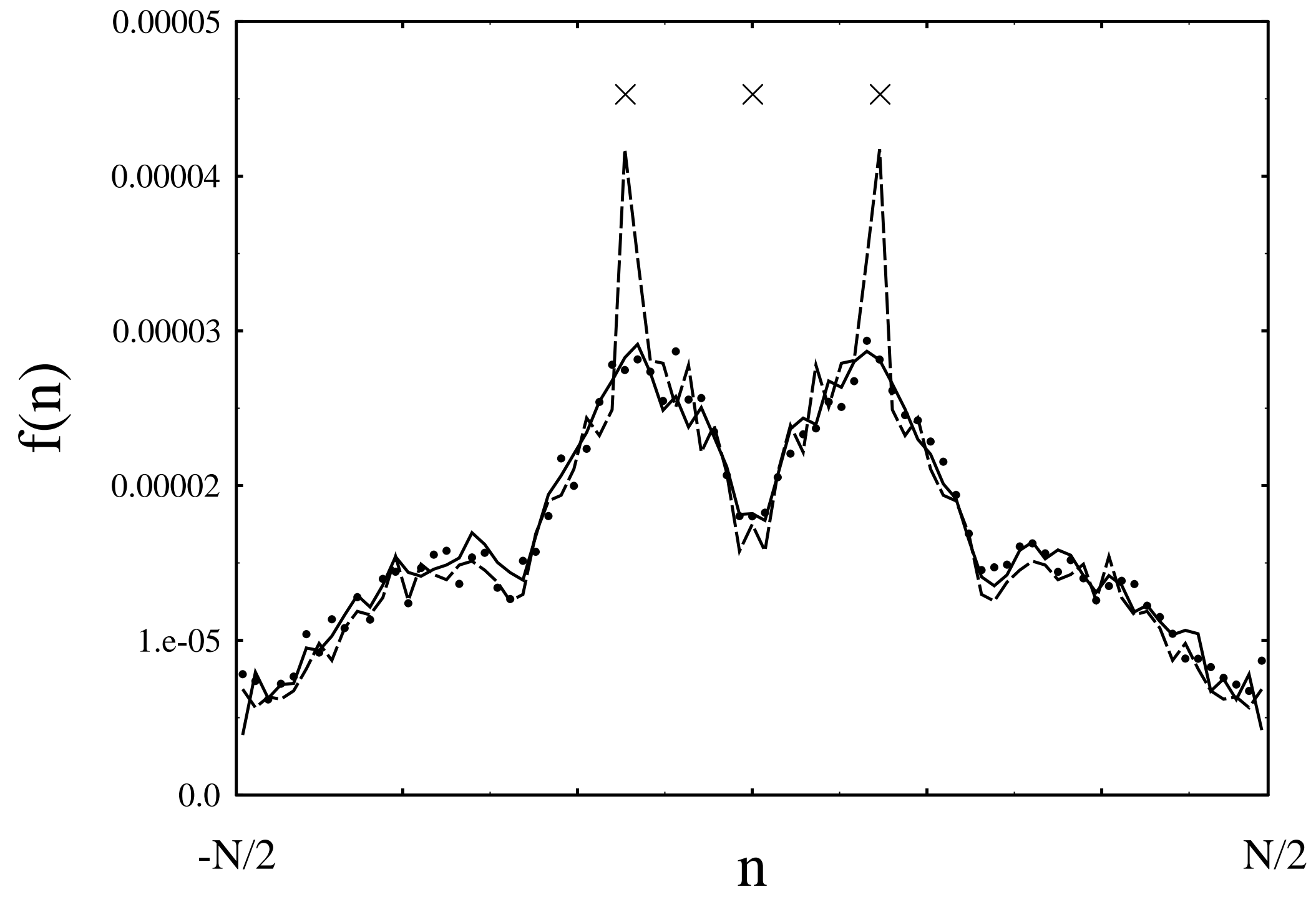

